\title{
The clinical usefulness of closed reduction of nasal bone using only a periosteal elevator with a rubber band
}

Young Ji Park, Woo Sang Ryu, Gyu Hyeon Kwon, Kyung Suk Lee

Department of Plastic and Reconstructive Surgery, Gyeongsang National University Hospital, Jinju, Korea
Background: Closed reduction of nasal fracture with various instrument is performed to treat nasal fracture. Depending on the type of nasal fracture and the situation in which it is being operated, the surgeon will determine the surgical tool. The objective of this study was to investigate whether a periosteal elevator (PE) was a proper device to perform closed reduction for patients with simple nasal fractures.

Methods: From March 2018 to December 2018, 50 cases of simple nasal bone fracture underwent closed reduction performed by a single surgeon. These patients were divided into two groups randomly: nasal bone reduction was performed using only PE (freer) and nasal bone reduction was performed using Walsham, Asch forcep, and Boies elevator (non-freer, non-PE).

Results: The paranasal sinus computed tomography was performed on patients before and after operation to carry out an accurate measurement of reduction distance at the same level. According to the results, the interaction between instruments and fracture types had a significant influence on reduction distance $(p=0.021)$. To be specific, reduction distance was significantly $(p=0.004)$ increased by $2.157 \mathrm{~mm}$ when PE was used to treat patients with partial displacement compared to that when non-PEs were used.

Conclusion: Closed reduction using PE and other elevator is generally an effective treatment for nasal fracture. In partial-displacement type of simple nasal fracture, closed reduction using PE can have considerable success in comparison with using classic instruments.

Keywords: Facial bone / Fracture / Nasal bone / Reduction / Treatment outcome

\section{INTRODUCTION}

The nose is the most protruding part of the face, located at the anatomical center of view. For this reason, the nose is most vulnerable to facial injuries. It is also aesthetically important. Nasal bone fracture anteriorly positioned at the center of the face is one of the most common fractures of the facial bone [1-3]. Na-

\section{Correspondence: Kyung Suk Lee}

Department of Plastic and Reconstructive Surgery Gyeongsang National University

Hospital, 79 Gangnam-ro, Jinju 52727, Korea

E-mail: opensound@hanmail.net

Received August 9, 2019 / Revised September 16, 2019 / Accepted September 17, 2019 sal bone fracture is a three-dimensional (3D) displacement that requires $3 \mathrm{D}$ reconstruction using various instruments.

Depending on the type of nasal fracture and the situation in which surgery is being conducted, the surgeon will determine which surgical tools to use [4-8]. Walsham forceps, Asch forceps, and Boies elevators are widely known surgical instruments that are inserted into nasal openings to lift the fracture site for reduction. Patients with complex nasal fractures and those with definite depression points are more likely to be corrected with these tools. However, fractures that are difficult to correct using relatively large tools at small gaps between a frac- 
tured bone and septum or small depressed point may be corrected using small instruments with minimal effort. Although the Boies elevator is thin and the width is similar to the size of the lateral nasal bones, its tips are blunt and relatively thick, making it difficult to correct small fractures.

Therefore, we used a periosteal elevator (PE) with a sharper, smoother tip and a smaller width than the Boies elevator so that it could be inserted into the small gap between the nasal bone and the nasal septum. The purpose of this study was to determine if PEs were more useful for correcting fractures in patients with relatively simple and small nasal bone fractures than conventional instruments.

\section{METHODS}

\section{Patients}

The present study was randomly conducted on patients $(n=50$, 28 males and 22 females; mean age, 39 years) who underwent closed reductions of nasal bone fractures (less than 2 weeks after trauma) from March 2018 to December 2018 performed by s single surgeon. Exclusion criteria were patients with nose trauma history, surgical history on the nose, multiple fractures or comminuted-type fractures, and septal deviation or severe displacement. Patients was classified by three factors: the degree of displacement (total displacement or partial-displacement), the fracture type (unilateral or bilateral), and the surgical instrument used (PE or non-PE) (Table 1). Total displacement was defined as complete separation of bone cross-section. Partial-displacement was defined as incomplete separation of bone cross-section. To investigate the association of distance reduction and the type of surgical instrument used, patients were divided into the total displacement patient group and the partial- displacement patient group. They were further divided into the PE subgroup that used PE (freer) and the non-PE subgroup, where a different elevator was used (non-freer). The computed tomography (CT) imaging results were used to compare lengths from the nasal septum to the fracture site at the same level before and after surgery between groups and subgroups.

\section{Surgical procedure}

Under general anesthesia, all patients underwent closed reduction of their nasal bone fractures by the same surgeon. Patients were divided into two groups randomly. In the PE subgroup, nasal bone reduction was performed using only PEs (Freer Septum Elevator, $18.4 \mathrm{~cm}$; Codman \& Shurtleff Inc., Raynham, MA, USA) where a $5 \mathrm{~cm}$ lengthening 7-French sized rubber band was placed at the end of the PE. The author applied a 2 $\mathrm{mm}$ thin and $4 \mathrm{~mm}$ wide rubber band to the tip of the PE (Fig. 1). Nasal bone reduction was performed in the non-PE subgroup using Walsham forceps, Asch forceps, and Boies elevators (non-PE). Before surgery, gauze containing 1\% lidocaine

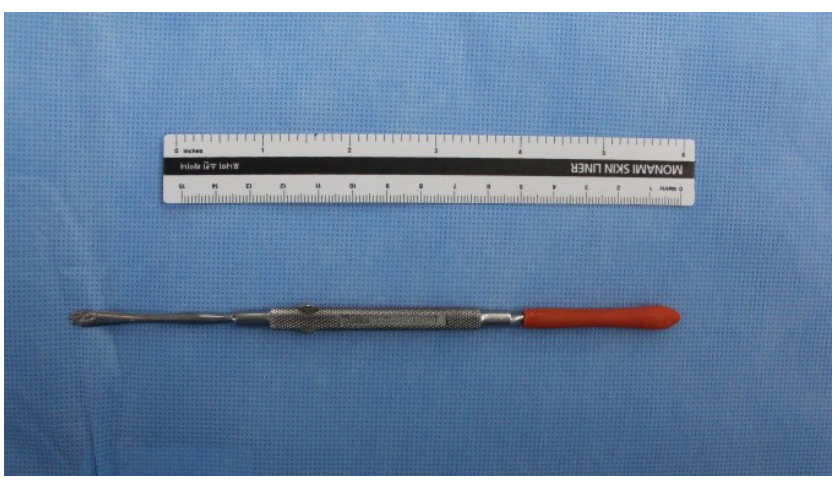

Fig. 1. A periosteal elevator with a rubber band applied at the end having $2 \mathrm{~mm}$ thin and $4 \mathrm{~mm}$ wide tip.

Table 1. Demographic and surgical characteristics of patients with nasal bone fracture

\begin{tabular}{|c|c|c|c|c|c|c|c|}
\hline \multirow{2}{*}{ Characteristic } & \multirow{2}{*}{ Total $(n=50)$} & \multicolumn{3}{|c|}{ Total displacement } & \multicolumn{3}{|c|}{ Partial displacement } \\
\hline & & $P E(n=10)$ & Non-PE $(n=7)$ & $p$-value & $P E(n=17)$ & Non-PE $(n=16)$ & $p$-value \\
\hline Age (yr) & $39.00 \pm 13.00$ & $40.50 \pm 18.50$ & $35.00 \pm 11.00$ & $0.810^{\mathrm{a})}$ & $39.00 \pm 4.00$ & $38.50 \pm 12.00$ & $0.569^{\text {a) }}$ \\
\hline Sex & & & & $0.644^{\text {b) }}$ & & & $0.732^{b)}$ \\
\hline Male & 28 & 7 & 4 & & 8 & 9 & \\
\hline Female & 22 & 3 & 3 & & 9 & 7 & \\
\hline Laterality & & & & $0.134^{\mathrm{b})}$ & & & $0.728^{\mathrm{b})}$ \\
\hline Unilateral & 31 & 4 & 6 & & 10 & 11 & \\
\hline Bilateral & 19 & 6 & 1 & & 7 & 5 & \\
\hline Distance from septum to fracture site (preoperative), $\mathrm{mm}$ & $2.80 \pm 0.40$ & $2.50 \pm 0.50$ & $3.20 \pm 0.70$ & $0.298^{\mathrm{a})}$ & $3.10 \pm 0.60$ & $3.30 \pm 0.50$ & $0.986^{\mathrm{a})}$ \\
\hline Distance from septum to nasal bone (postoperative), mm & $5.90 \pm 1.00$ & $5.15 \pm 0.35$ & $5.80 \pm 0.40$ & $0.222^{\mathrm{a})}$ & $7.50 \pm 0.70$ & $5.60 \pm 1.20$ & $0.037^{\mathrm{a}, \mathrm{c}, \mathrm{c}}$ \\
\hline Reduction distance (postoperative-preoperative), mm & $2.60 \pm 0.40$ & $2.45 \pm 0.15$ & $2.60 \pm 0.40$ & $0.923^{\text {a) }}$ & $4.40 \pm 0.60$ & $2.15 \pm 1.40$ & $<0.001^{\mathrm{a}, \mathrm{c})}$ \\
\hline
\end{tabular}

Values are presented as mean $\pm S D$ or number.

$\mathrm{PE}$, periosteal elevator.

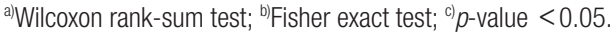


and 1:100,000 epinephrine was packed in the nasal cavity to reduce bleeding. The fracture site of the nasal dorsum indicated by physical examination, plain radiograph, and orbital 3D CT was marked with a marking pen (Fig. 2). After 15 minutes, the author positioned the elevator right below the fracture site and lifted it laterally or superiorly. After continuity and symmetry of the nasal bone was identified using fingers, we confirmed the endpoint of nasal reduction. Upon reduction, Merocel (Fabco Inc., London, UK) was packed into the nasal cavity below the fracture site and an external splint (Megen Inc., Seoul, Korea) was applied for protection.

\section{Assessment of postoperative results}

Postoperative CT outcomes were compared to preoperative and immediately postoperative paranasal sinus CTs. Before closed reduction of nasal bone, the horizontal distance from the innermost point of the nasal fracture to the septum was measured on axial view CT. After surgery, the distance was measured again at the same level of the axial view CT. After that, the author checked the horizontal distance from the corrected innermost part of the nasal bone to the septum (Fig. 3).

\section{Statistical analyses}

Descriptive statistics, including the median, interquartile range, frequency, and percentage, were used to summarize the variables. The nonparametric Wilcoxon rank-sum test for continuous variables, such as age or reduction distance, and Fisher exact test for binary variables, such as sex or laterality, were used
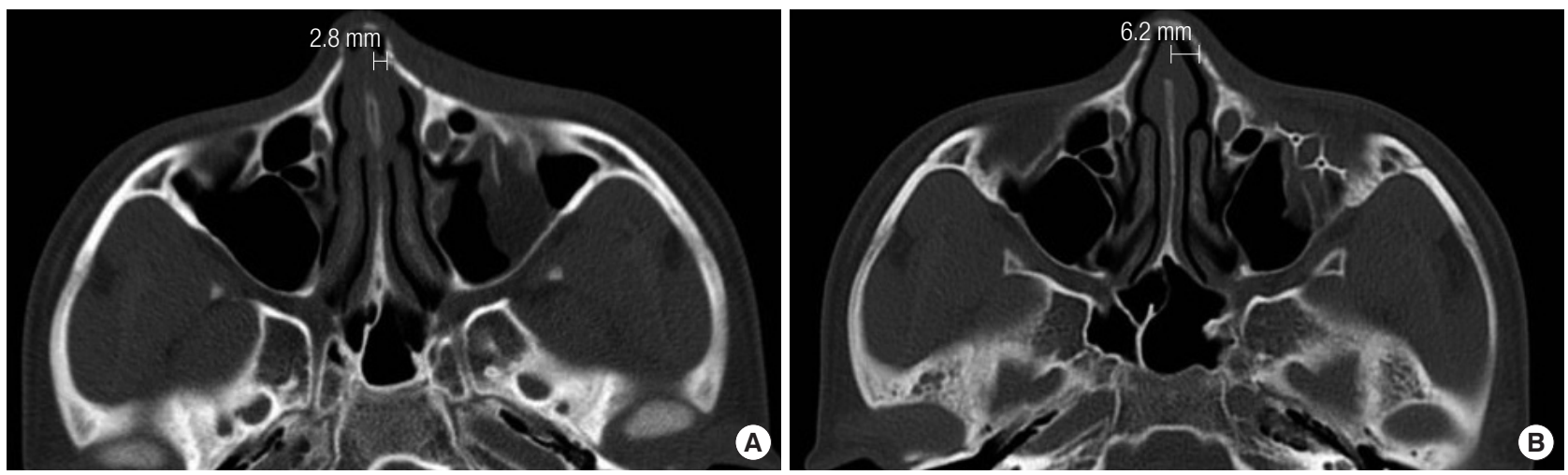

Fig. 2. (A) Before closed reduction, the distance from the septum to the fracture site was measured by computed tomography (2.8 mm). (B) The distance after surgery was measured again to confirm the degree of reduction $(6.2 \mathrm{~mm})$.
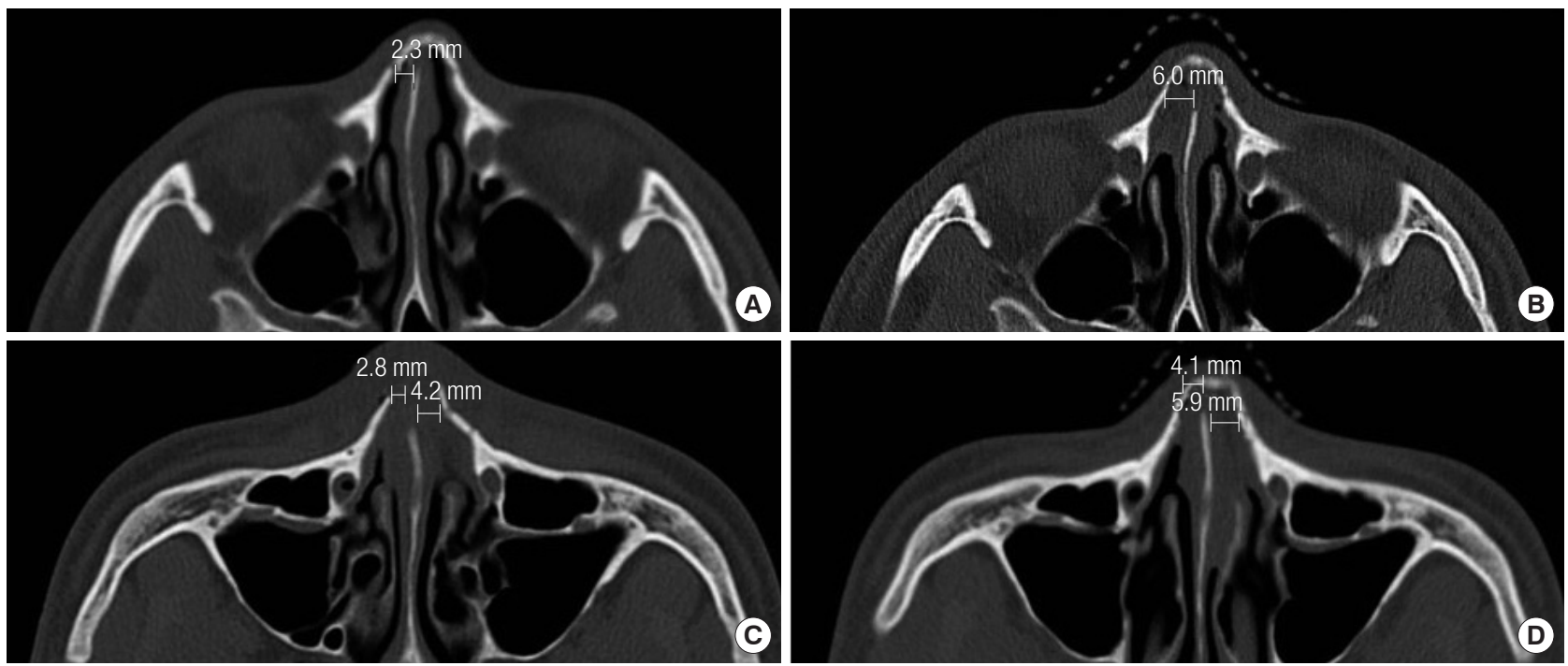

Fig. 3. (A) A 35-year-old male who slipped down 2 days ago visited the clinic with right unilateral nasal bone fracture. (B) After closed reduction of nasal bone using freer, the paranasal sinus computed tomography (PNS CT) was taken immediately and the distance of reduction was measured (from $2.3 \mathrm{~mm}$ to $6.0 \mathrm{~mm}$ ). (C) A 54-year-old male who hit elbow 5 days ago visited the clinic with bilateral nasal bone fracture. (D) After closed reduction of nasal bone using Asch forceps, the PNS CT was taken immediately and the distance of reduction was measured (from $2.8-4.2 \mathrm{~mm}$ to $4.1-5.9 \mathrm{~mm}$ ). 


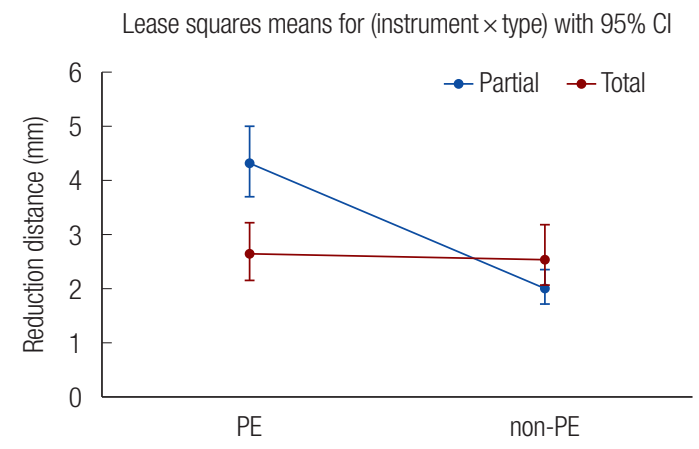

Fig. 4. Reduction distance according to instrument and location. $\mathrm{PE}$, periosteal elevator; Partial, partial displacement; Total, total displacement; CI, confidence interval.

to compare values between the two groups (the PE group and the non-PE group). Multiple linear regression with the enter method was conducted to examine the association between distance reduction and the surgical instruments (instrument) used according to nasal bone fracture type (type) with its interaction term between instrument and type (instrument $\times$ type), after adjusting for sex, age, and laterality. Before the multiple linear regression analysis, logarithmic transformation of the reduction distance variable was conducted to improve normality of data. We estimated least-squares means of the interaction term in the model and then presented the value with $95 \%$ confidence interval (Fig. 4). Bonferroni correction was applied for multiple comparisons in the post-hoc analysis. Statistical significance was set at $5 \%$. All statistical analyses were conducted using SAS 9.4 (SAS Institute, Cary, NC, USA).

\section{RESULTS}

A total of 50 patients ( 28 males and 22 females) with nasal bone fractures and a mean age of 39 years were included in the study. There was no statistically significant difference in age, sex, or laterality between groups using the Wilcoxon signed-rank test or Fisher exact test. In the total displacement patient group, mean preoperative distances from the nasal septum to the fracture site in the PE subgroup and the non-PE subgroups were 2.50 and $3.20 \mathrm{~mm}$, respectively. After surgery, reduction distances in these two groups were 5.15 and $5.80 \mathrm{~mm}$, respectively. In the partial-displacement patient group, mean preoperative distance from the nasal septum to the fracture site in PE and non-PE subgroups were 3.10 and $3.30 \mathrm{~mm}$, respectively, whereas reduction distances in these two subgroups after surgery were 7.50 and $5.60 \mathrm{~mm}$, respectively (Table 1).

In the total displacement group, when the distance from the septum to the nasal bone fracture before and after surgery was compared between PE and non-PE subgroups using the Wil- coxon signed-rank test, there was no statistically significant difference. However, in the partial-displacement group, when the postoperative distance from the septum to the nasal bone fracture was compared between PE and non-PE subgroups, there was a statistically significant difference $(p=0.037)$ (Table 1$)$.

In the total displacement group, there was no statistically significant difference in postoperative or preoperative distances between subgroups. However, in the partial-displacement group, there was a statistically significant difference in distance reduction between PE and non-PE subgroups $(p<0.001)$ (Table 1).

The interaction between surgical instruments and the type of fracture was examined using least-squares means. The results showed no significant difference according to the type of surgical instruments in the total displacement group, although the partial-displacement group showed significant differences depending on the type of surgical instrument used (Fig. 4).

\section{DISCUSSION}

Nasal bone fractures are very common fractures due to a variety of causes. Because of the variety of fractures and the extent of surgical instrument options, appropriate surgical treatment should be used. A study by Rohrich and Adams [3] of 110 cases has reported that preoperative evaluation of the nasal bone and nasal septum is very important, especially when primary reconstruction of the nasal septum is considered [9]. Therefore, if the nasal septum is severely displaced or bent, there are complex nasal bone fractures, or if the deformation is severe, it is better to use conventional instruments, such as Walsham and Ash forceps. As such, studies have been conducted on the proper timing of nasal bone fracture reduction or which instrument can most effectively reduce nasal bone fractures. However, there were limitations in applying conventional instruments to simple fractures. In addition, no specific study has been conducted on the effective reduction of simple fractures.

The author suggests that if there is a small fracture or a fracture that lies down at the tip of the nasal bone, an instrument like a PE can help when correction with conventional instruments is difficult. According to Hwang et al. [10], CT is essential for fracture diagnosis, not only for nasal bone fractures, but also for septal fractures because the reliability of simple X-ray is $82 \%$ [11]. Therefore, we divided patients into two groups, total displacement and partial-displacement and used PE and nonPE instruments, comparing the results on CT findings.

Since the intranasal area is significantly narrowed by an external injury, a sharp and pointed surgical instrument might be more efficiently used. The reduction distance was significantly different depending on the type of surgical instrument used 
and fracture type. The factor that had a significant influence on the partial-displacement group was the use of a PE. This means that delicate reduction techniques are needed in partial-fracture cases and a PE with a sharp and pointed tip, worked very well.

This study has some limitations. First, this study had a small number of patients, necessitating the use of various types of statistics for obtaining statistically significant results. If a larger number of samples are used in future studies, more reliable results will be obtained. In addition, this study was conducted on simple fractures. Thus, the usefulness of PE in complex or severe fractures was not determined.

Various attempts have been made to develop an effective treatment for nasal bone displacement. Based on results of this study, the authors believe that the surgical PE is more suitable than classical instruments to treat patients with partial-displacement and simple nasal bone fracture. It will be useful in the future.

\section{NOTES}

\section{Conflict of interest}

No potential conflict of interest relevant to this article was reported.

\section{Ethical approval}

The study was approved by the Institutional Review Board of Gyeongsang National University Hospital (IRB No. GNUH 2019-08-001) and performed in accordance with the principles of the Declaration of Helsinki. Written informed consents were obtained.

\section{Patient consent}

The patients provided written informed consent for the publication and the use of their images.

\section{ORCID}

Young Ji Park https://orcid.org/0000-0001-8416-7833
Woo Sang Ryu https://orcid.org/0000-0002-7472-3813

Gyu Hyeon Kwon https://orcid.org/0000-0002-8377-4698

Kyung Suk Lee https://orcid.org/0000-0001-7731-1761

\section{REFERENCES}

1. Chung SH, Park J, Choe J, Baek SM. Clinical analysis of satisfaction of nasal bone reduction. J Korean Soc Plast Reconstr Surg 1994;21:984-90.

2. Hong SB, Choi BW, Suh IS, Ha JH. Clinical \& radiological evaluation of the nasal bone fractures. J Korean Soc Plast Reconstr Surg 1996;23:1572-82.

3. Rohrich RJ, Adams WP Jr. Nasal fracture management: minimizing secondary nasal deformities. Plast Reconstr Surg 2000; 106:266-73.

4. Lee YJ, Lee KT, Pyon JK. Finger reduction of nasal bone fracture under local anesthesia: outcomes and patient reported satisfaction. Arch Craniofac Surg 2019;20:24-30.

5. Kang CM, Han DG. Objective outcomes of closed reduction according to the type of nasal bone fracture. Arch Craniofac Surg 2017;18:30-6.

6. Stranc MF, Robertson GA. A classification of injuries of the nasal skeleton. Ann Plast Surg 1979;2:468-74.

7. Pollock RA. Nasal trauma: pathomechanics and surgical management of acute injuries. Clin Plast Surg 1992;19:133-47.

8. Ridder GJ, Boedeker CC, Fradis M, Schipper J. Technique and timing for closed reduction of isolated nasal fractures: a retrospective study. Ear Nose Throat J 2002;81:49-54.

9. Mondin V, Rinaldo A, Ferlito A. Management of nasal bone fractures. Am J Otolaryngol 2005;26:181-5.

10. Hwang K, You SH, Kim SG, Lee SI. Analysis of nasal bone fractures; a six-year study of 503 patients. J Craniofac Surg 2006; 17:261-4.

11. Rhee SC, Kim YK, Cha JH, Kang SR, Park HS. Septal fracture in simple nasal bone fracture. Plast Reconstr Surg 2004;113:4552. 